Conclusion This results confirm the presence of an increased number of lymphatic vessels in RA synovial tissue. Moreover, the authors observed D2-40+ structures, mostly around preexisting blood vessels, indicating the development of a new lymphatic network in the synovial sublining. The presence of D2-40+ cells in the inflammatory infiltrate and in the macrophage-like synoviocites, lead us to hypothesise that in RA, macrophages are activated and may function as lymphatic precursors. In RA synovium, both JAM-A and JAM-C are expressed in all D2-40+ cells, indicating their participation in the inflammatory process and intercellular junction assembly. Their expression in new lymphatic vessels and in D2-40+ cells stimulated to form new vessels, confirm their involvement in the lymphoangiogenic process occurring in RA.

\title{
A130 INVOLVEMENT OF JUNCTIONAL ADHESION MOLECULES IN LYMPHATIC VASCULAR REMODELLING IN RHEUMATOID ARTHRITIS
}

A F Milia, ${ }^{1}$ M Manetti, ${ }^{2}$ S Pfanner, ${ }^{3}$ F Nacci, ${ }^{1}$ G Fiori, ${ }^{1}$ S Guiducci, ${ }^{1}$ E Romano, ${ }^{1}$ C Ceccarelli, ${ }^{1}$ L Ibba-Manneschi, ${ }^{2}$ M Matucci-Cerinic ${ }^{1}$ Biomedicine, Division of Rheumatology, University of Florence, Unit of Surgery of the Hand and Microsurgery, CTO, Florence, Italy; ${ }^{2}$ Anatomy, Histology and Forensic Medicine, University of Florence; ${ }^{3}$ Unit of Surgery of the hand and microsurgery, CTO, Florence, Italy

\subsection{6/ard.2010.148999.6}

Background and objective Rheumatoid arthritis (RA) is a chronic systemic autoimmune disease, in which neovascularisation of the synovium is regarded as a crucial step in its development and progression. Lymphatic neovascularisation has been observed in RA joints. Junctional adhesion molecule A (JAM-A) and JAM-C regulate leucocyte-endothelial cell (EC) interactions. JAMs also interact at inter-EC junctions regulating vascular permeability and are implicated in pathophysiological processes involving leukocyte transmigration, tight junction assembly and angiogenesis.

The aim of this study was to investigate the role of JAM-A and JAM-C in mediating the lymphoangiogenic process in RA synovium.

Materials and methods Synovial biopsies from RA and osteoarthritic (OA) patients, used as control, has been collected during arthroplasty or synovectomy. Synovial sections were stained with primary antibodies (Abs) antihuman JAM-A or JAM-C followed by fluorochrome-conjugated secondary Abs. The lymphatic EC specific marker podoplanin (D2-40) was used to differentiate blood (D2-40-) and lymphatic (D2-40+) vessels.

Results Immunohistochemical analysis showed, in RA synovium, the presence of abundant D2-40+ structures, identifiable as lymphatic vessels. Many D2-40+ cells were also found in the intimal lyning layer, specially in the hyperplasic areas. Lymphatic vessels were distributed in the sublining, specially around blood vessels. Clusters of D2-40+ cells were also found in the inflammatory infiltrate, in structures resembling new lymphatic vessels. In all sections from RA, JAM-A and JAM-C were observed on almost all vasculature including the lymphatic (D2-40+) and blood vessels (D2-40-). Initial lymphatic vessels reacted with anti-JAM-A and anti-JAM-C. In the synovial lining, JAM-A and JAM-C were expressed on D2-40+ macrophage-like synoviocites. D2-40+ cells in the inflammatory infiltrate showed positivity for both JAM-A and JAM-C. The immunostaining for both JAMs was weaker in OA than in RA synovium. 